\title{
A journey from the Physical Web to the Physical Semantic Web*
}

\author{
Michele Ruta \\ michele.ruta@poliba.it \\ Polytechnic University of Bari \\ Bari, Italy \\ Filippo Gramegna \\ filippo.gramegna@poliba.it \\ Polytechnic University of Bari \\ Bari, Italy
}

\author{
Floriano Scioscia \\ floriano.scioscia@poliba.it \\ Polytechnic University of Bari \\ Bari, Italy \\ Saverio Ieva \\ saverio.ieva@poliba.it \\ Polytechnic University of Bari \\ Bari, Italy
Eugenio Di Sciascio
eugenio.disciascio@poliba.it
Polytechnic University of Bari
Bari, Italy

\author{
Giuseppe Loseto \\ giuseppe.loseto@poliba.it \\ Polytechnic University of Bari \\ Bari, Italy \\ Agnese Pinto \\ agnese.pinto@poliba.it \\ Polytechnic University of Bari \\ Bari, Italy
}

\begin{abstract}
The Physical Semantic Web (PSW) is a novel paradigm built upon the Google Physical Web (PW) approach and devoted to improve the quality of interactions in the Web of Things. Beacons expose semantic annotations instead of basic identifiers, i.e., machineunderstandable descriptions of physical resources. This enables novel ontology-based object advertisement and discovery and in turn- advanced user-to-thing and autonomous thing-to-thing interactions.

The demo shows the evolution from the PW to the PSW in a discovery scenario set in a winery, where bottles are equipped with Bluetooth Low Energy beacons and a customer can discover them using her smartphone. The final goal is to prove benefits of PSW over basic PW, including: rich semantic-based object annotation; dynamic annotations exploiting on-board sensors; enhanced discovery and ranking of nearby objects through semantic matchmaking; availability of interactions even without working Internet infrastructure, by means of point-to-point data exchanges.
\end{abstract}

\section{CCS CONCEPTS}

- Information systems $\rightarrow$ Web Ontology Language (OWL); • Human-centered computing $\rightarrow$ Ubiquitous and mobile computing; • Computing methodologies $\rightarrow$ Knowledge representation and reasoning;

\section{KEYWORDS}

Semantic Web of Things, Physical Web, Resource discovery, Nonstandard reasoning

"Produces the permission block, and copyright information

This paper is published under the Creative Commons Attribution 4.0 International (CC BY 4.0) license. Authors reserve their rights to disseminate the work on their personal and corporate Web sites with the appropriate attribution.

WWW' 18 Companion, April 23-27, 2018, Lyon, France

( 2018 IW3C2 (International World Wide Web Conference Committee), published under Creative Commons CC BY 4.0 License.

ACM ISBN 978-1-4503-5640-4/18/04.

https://doi.org/10.1145/3184558.3186981

\section{ACM Reference Format:}

Michele Ruta, Floriano Scioscia, Giuseppe Loseto, Filippo Gramegna, Saverio Ieva, Agnese Pinto, and Eugenio Di Sciascio. 2018. A journey from the Physical Web to the Physical Semantic Web. In The 2018 Web Conference Companion, April 23-27, 2018, Lyon, France. ACM, New York, NY, USA, 4 pages. https://doi.org/10.1145/3184558.3186981

\section{INTRODUCTION}

The Physical Web ${ }^{1}$ (PW) [5] is a framework for the Internet of Things (IoT), proposed by Google Inc. and devoted to implement direct users-to-things interactions. This paradigm is based on resource discovery enabling mobile personal devices (e.g., smartphones) to retrieve Uniform Resource Locators (URLs) of nearby objects through the Eddystone Bluetooth beacon protocol ${ }^{2}$. Finding URLs via Wi-Fi through multicast DNS (mDNS) and Universal Plug and Play (uPnP) is also supported. The approach does not require directory services, as smart objects are associated to unambiguous Web addresses.

Although such an approach promotes human-to-thing communication (providing indubitable benefits to end users from a direct interaction with pervasive computing contexts), there are some significant issues penalizing a full adoption of the Google PW framework in advanced applications. Particularly: i) apart from the early Bluetooth-based discovery, further interactions require a mandatory Internet connection to the Web; ii) direct thing-tothing communication is not allowed yet; iii) discovery mechanisms are too simplistic to cope with typical interoperability and unpredictability problems in the IoT. The Physical Semantic Web (PSW) [2] proposal improves upon the PW by associating semantic annotations to object beacons instead of trivial identifiers, i.e., machineunderstandable descriptions in standard Semantic Web languages. Therefore object advertisement and discovery are enhanced and things can communicate among them through proper software agents without user mediation.

The PSW approach preserves the original Eddystone-URL beacons discovery, so legacy applications are still supported. Furthermore, in the PSW each URL could alternatively target: i) a basic Web

\footnotetext{
${ }^{1}$ https://google.github.io/physical-web/

${ }^{2} \mathrm{http} / /$ github.com/google/eddystone
} 


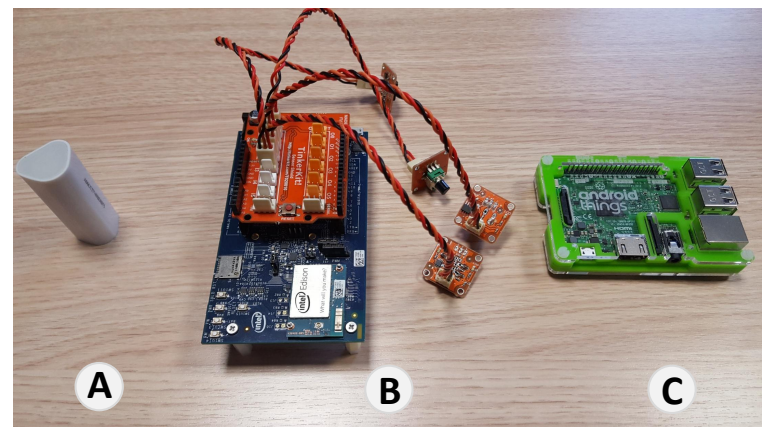

Figure 1: Demo beacons

page; ii) an annotated Web page, where users may view contents and/or exploit metadata semantics; iii) a semantic annotation to be directly processed by software agents locally.

Object discovery ensues from a semantic matchmaking process [3], able to compare a request (i.e., user or object profile) with several beacon annotations, referred to the same ontology. For each 〈user/object profile, resource〉 pair, a matchmaking score measures the relevance of the retrieved beacon w.r.t. request annotation. Concept Abduction and Contraction non-standard inferences [3] theoretically ground the PSW discovery and provide also an outcome explanation capability: it evidences compatible, incompatible and missing elements, so as to eventually refine the original query.

The proposed demo shows the evolution from the PW to the PSW in a four-step experience, as outlined in Section 2. The reference scenario is set in a winery, where bottles available for sale are equipped with Bluetooth Low Energy (BLE) beacons and a customer can discover them using a smartphone. Discussion in Section 3 highlights how relevant elements of the demo setup help understand both potentialities and benefits of the PSW proposal if compared with standard PW.

\section{DEMO DESCRIPTION}

The demonstration consists of a mobile application, showcasing the most significant PSW features. It can be conceptually divided in four stages, each described in the following subsections. Customers in a winery use their smartphones to discover bottles equipped with $\mathrm{PW} / \mathrm{PSW}$ beacons. In each stage the user exploits progressively even more advanced functions, including the simulation of variations in environmental parameters (conditioning the discovery). For the purposes of demonstrating the app, a PSW-compatible Android smartphone $^{3}$ is used. Bottles are equipped with two standard PW beacons like the Bluvision BEEKs Lite ${ }^{4}$ shown in Figure 1(A) and three PSW beacons (two PSW-URL and one PSW-UID), represented by two Intel Edison ${ }^{5}$ boards like in Figure 1(B) and a Raspberry Pi 3 Model $B^{6}$ like in Figure $1(\mathrm{C})$. Generic Wi-Fi Internet connectivity and energy supply are the only requirements for the demo environment; a monitor can be used to replicate the smartphone screen for greater visibility and convenience.

\footnotetext{
${ }^{3}$ Required Android API level 21, corresponding to operating system version 5.0. ${ }^{4}$ https://shop.bluvision.com/products/beeks-lite

${ }^{5} \mathrm{http}: / /$ software.intel.com/en-us/iot/hardware/edison/documentation

${ }^{6}$ https://www.raspberrypi.org/products/raspberry-pi-3-model-b/
}

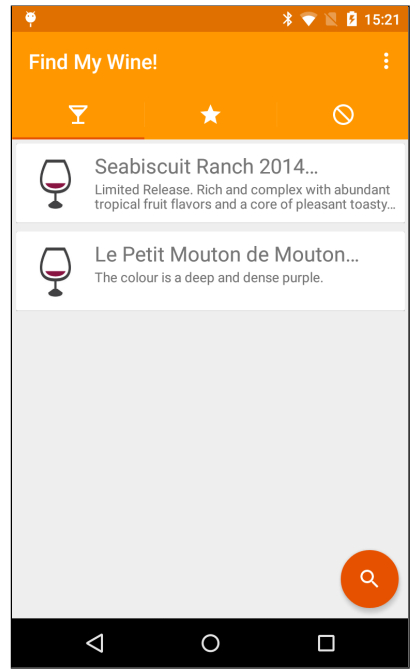

(a) Beacons List

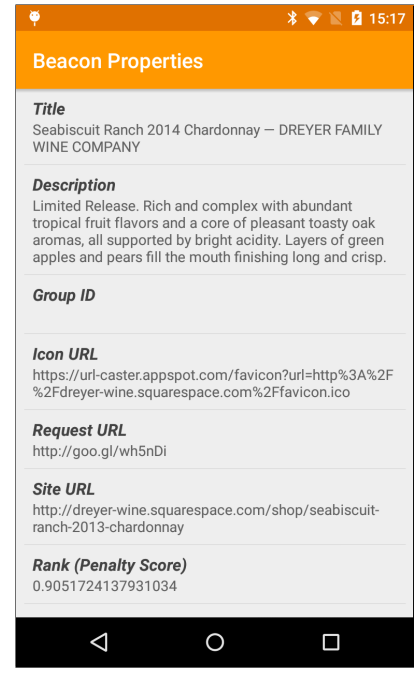

(b) Beacon Info
Figure 2: Stage 1 - screenshots of the Android client

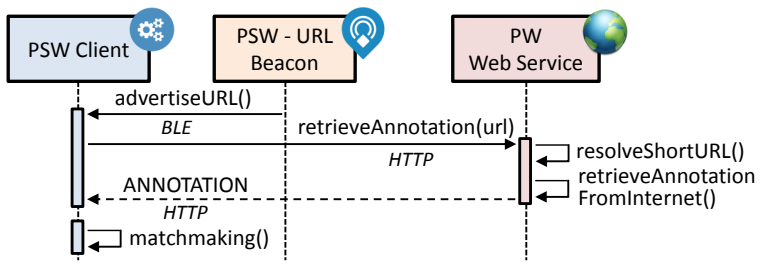

(a) Eddystone-PSW-URL beacon

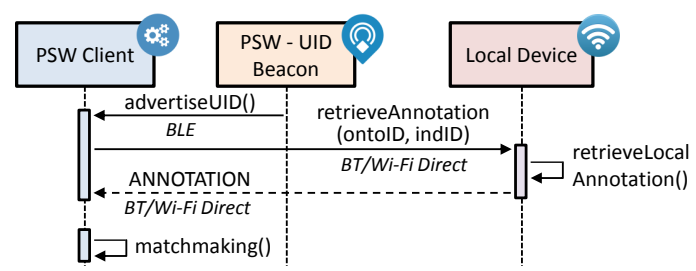

(b) Eddystone-PSW-UID beacon

Figure 3: Discovery of PSW Eddystone beacons

\subsection{Stage 1: Physical Web}

Francesco is organizing an elegant dinner party. He enters a winery looking for a fine bottle. After activating Bluetooth on his smartphone, he starts the Find My Wine! Android application to discover the exposed beacon advertisements. Detected ads are listed in a result screen, as shown in Figure 2(a). At this stage, only two Physical Web beacons are visible in the cellar. They expose URLs to the specific wine Web pages and are ordered according to the physical distance from the user (estimated by comparing received signal strength and transmitted power field in Eddystone frames).

For each beacon, the app can retrieve some basic metadata (e.g., title/description as in Figure 2(b)) or open the advertised Web page in the browser. All beacon information is retrieved by resolving the 


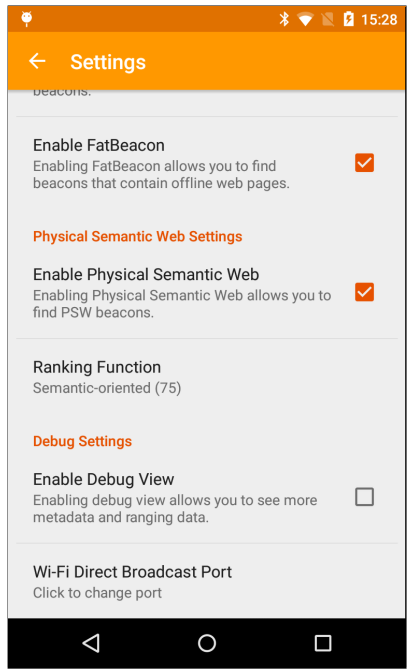

(a) Settings

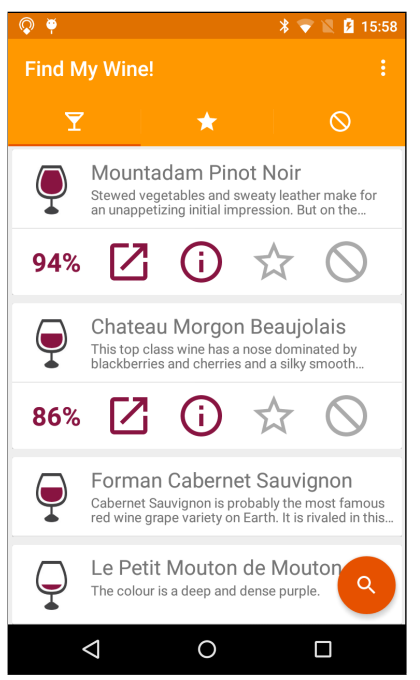

(c) Detected PSW beacons

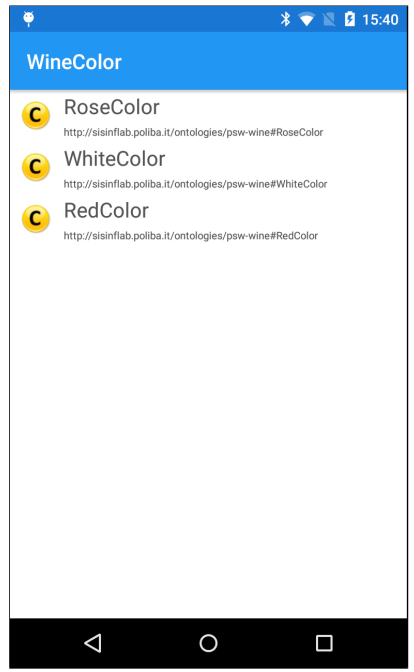

(b) Request editor

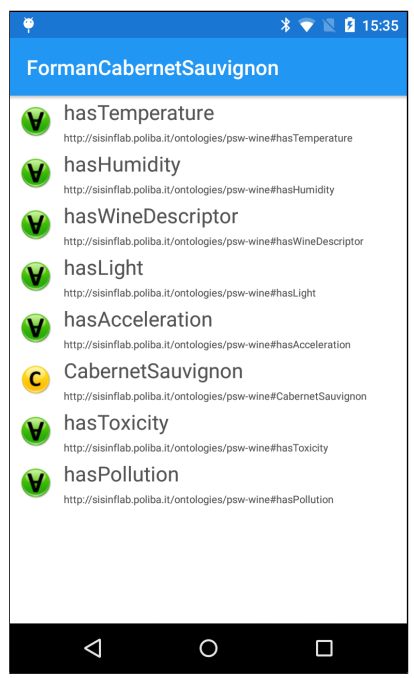

(d) Annotation viewer
Figure 4: Stage 2 - screenshots of the Android client

advertised Eddystone URL with a remote Physical Web Service ${ }^{7}$ via HTTP.

\subsection{Stage 2: Physical Semantic Web}

The user can enable the Physical Semantic Web option from the settings screen in Figure 4(a) to perform a more accurate and flexible resource discovery ${ }^{8}$. In addition to previously discovered wines, three PSW beacons (specifically, two PSW-URL and one PSW-UID) are advertised now through Intel Edison or Raspberry Pi 3 boards

\footnotetext{
${ }^{7}$ https:/github.com/google/physical-web/tree/master/web-service

${ }^{8}$ Typically, PSW support should be always on, given the backward compatibility of the proposed PW enhancements. Anyway, the user option has been implemented for demo purposes in order to make more evident discovery differences switching from PW-only mode to PW+PSW mode and back.
}

running the PSW-toolkit framework ${ }^{9}$. Each PSW-URL beacon exposes a URL used to download a Web Ontology Language [4] annotation from the Web (Figure 3(a)), whereas the PSW-UID beacon advertises an OWL annotation stored on the beacon itself. In the last case, as shown in Figure 3(b), the user device retrieves the resource description through a peer-to-peer connection (e.g., Bluetooth) [2].

User's mobile device embeds a semantic matchmaking engine [3] allowing to rank retrieved annotations in relevance order w.r.t. a request. Requests can be either built implicitly -by means of context-aware profiler daemons running on the device- or explicitly, through e.g., a graphical or speech-based user interface. The demo app provides a graphical editor of OWL requests to: i) navigate the reference taxonomy describing main wine features (e.g., wine colors in Figure 4(b)); ii) show both class and object property hierarchy; iii) compose a request selecting basic OWL ontology elements. For the purposes of the demo, a simple reference ontology has been created, inspired by classical examples in [1]. OWL annotations of bottles contain both a description of the wine and conservation status information. Beacons are ranked w.r.t. the user request by means of a semantic-based utility function [2]. The adopted semantic matchmaking relies on non-standard inference services in Description Logics. Whenever the request is incompatible with an object's annotation (i.e., their conjunction is unsatisfiable), Concept Contraction determines what part of the request cause the incompatibility and therefore should be retracted to pursue a match. If user's request and object's annotation are compatible, but the latter does not contain all requested features, Concept Abduction identifies the request elements not explicitly covered and therefore to be hypothesized about the object (under Open World Assumption) to reach a full match. As detailed in [3], penalty functions are associated to the outcome of Concept Contraction and Abduction -since minimal retraction and hypothesis are preferred-and provide a semantic distance metric of a set of objects w.r.t. a given request, enabling a logic-based relevance ranking. In the app settings, several weight configurations are available to combine the semantic distance with context-aware data-oriented attributes (i.e., physical distance and user's preference) in the general penalty formula described in [2].

In case of trivial syntactic matching, all beacons would have the same score. A semantic-based matchmaking and a proper modelling of the reference knowledge base, make possible instead to highlight discrepancies between descriptions. By selecting a beacon, the user can: i) open in the browser the related Web page; ii) only for PSW devices, show the exposed OWL annotation (as in Figure 4(d)) and likewise the matchmaking outcome explanation. In the last case, the user can navigate missing and incompatible features between the bottle annotation and the user request. In Figure 4(c), Mountadam Pinot Noir obtains the highest rank because it covers all features specified by the user. Chateau Morgon Beaujolais and Forman Cabernet Sauvignon obtain a lower score because they cover the request partially and also contain inappropriate features.

\subsection{Stage 3: Advanced PSW features}

PSW supports OWL annotations exposed by (both PSW-URL and PSW-UID) beacons changing dynamically due to events/phenomena

\footnotetext{
${ }^{9}$ http://github.com/sisinflab-swot/physical-semantic-web
} 


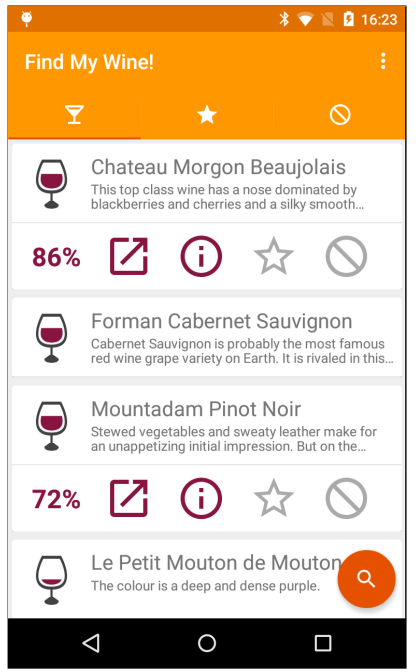

(a) Updated list

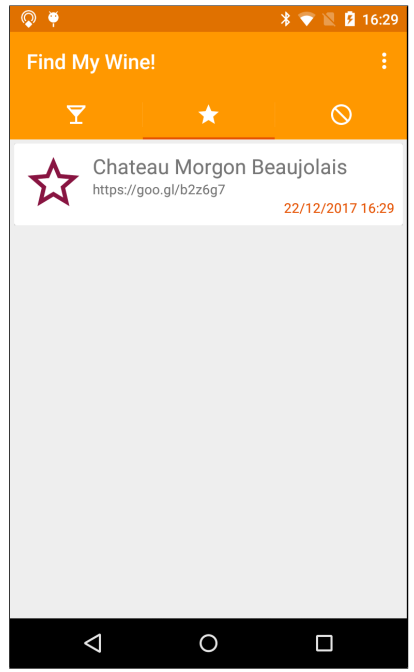

(b) Favorite beacons
Figure 5: Stage 3 - screenshots of the Android client

occurring to objects and/or context. In the proposed demo, the quality of each wine bottle can change according to storage conditions during its lifetime. An Arduino TinkerKit Sensor Shield is connected to one of the Intel Edison boards to emulate variations of environmental conditions in the winery, such as temperature, humidity and luminosity. Acting on the attached potentiometer, it is possible to simulate the situation of e.g., a bottle left for some hours in a place with a very low temperature, very high humidity and high luminosity. Due to this reason, the wine quality decreases and the related annotation is updated. When a new discovery round starts, degradation of the wine is detected, leading to a final rank lower than before: see in Figure 5(a) the case of modifications on Mountadam Pinot Noir.

A history list keeps already explored objects when the user opens URLs or displays OWL annotations. Moreover, the user can mark one or more beacons as favorites (i.e., useful, as in Figure 5(b)) or spam (i.e., useless or unwanted): in the following discovery procedures the rank of such beacons will be respectively increased or decreased.

\subsection{Stage 4: Infrastructureless PSW}

No phone networks signal is available in the winery cellar; owners provide Wi-Fi Internet connection in the showroom area but Francesco would like visit the basement. In the Physical Web, a connection is always required to retrieve data from the Web. In the PSW, exploiting Eddystone-UID frames, knowledge exchange can also occur through point-to-point connectivity. The proposed app demonstrates that all PSW features are available even when Wi-Fi and cellular data networks are switched off on the smartphone. Furthermore, since in IoT scenarios connectivity is often intermittent, PSW allows caching OWL annotations retrieved from detected objects for a limited time.

\section{DISCUSSION}

The proposed demo provides interactive examples of the benefits of the Physical Semantic Web over the basic Physical Web framework. It is conceived as a four-stage journey, to engage users with various level of expertise and knowledge on the specific technologies involved. The reference scenario has been chosen to be easy to understand.

The first stage is dedicated to the current Physical Web paradigm. By trying out the basic PW discovery features, even users with no prior working knowledge of PW solutions can familiarize with the benefits of the general PW proposal as well as with its limits. The second stage introduces the PSW framework and allows an interactive first-hand experience of the enhancements to the overall capabilities. In particular, users should be able to appreciate the benefits of fine-grained matchmaking enabled by accurate semanticbased object annotation. The third stage demonstrates advanced features, such as favorite and spam caching, as well as the PSW ability to deal with dynamic annotations. In this case, users are able to set input data directly, by adjusting potentiometers attached to the demo PSW beacon.

Finally, the fourth and last demo stage shows how the PSW grants interaction even when a network infrastructure is unavailable, by exploiting point-to-point data exchanges only. This may appear as a corner case in the reference demo scenario, although most wineries are actually located in cellars without cellular or wireless local area network connectivity. Most importantly, however, that kind of situations is frequent in challenging IoT application scenarios (e.g., environmental monitoring, surveillance, robot search and rescue teams, etc.), where stable Web connectivity cannot be granted. In those cases, basic interactions based on data retrieved from remote web services (e.g., through simple URLs associated to beacons or barcodes) result unusable. The PSW still supports full capabilities, so enabling a whole new range of thing-to-thing cooperation scenarios through on-board autonomous software agents in addition to userto-thing interactions.

A Web page ${ }^{10}$ describing the proposed demo is made available. It contains further screenshots of the mobile application, links to the source code and the ontology and a sample demo screencast.

\section{REFERENCES}

[1] Natalya F Noy and Deborah L McGuinness. 2001. Ontology development 101: A guide to creating your first ontology. Technical Report. Stanford knowledge systems laboratory tech. rep. KSL-01-05 and Stanford medical informatics tech. rep. SMI-2001-0880.

[2] Michele Ruta, Floriano Scioscia, Saverio Ieva, Giuseppe Loseto, Filippo Gramegna, and Agnese Pinto. 2017. Knowledge Discovery and Sharing in the IoT: The Physical Semantic Web Vision. In Proceedings of the Symposium on Applied Computing (SAC '17). ACM, New York, NY, USA, 492-498.

[3] Floriano Scioscia, Michele Ruta, Giuseppe Loseto, Filippo Gramegna, Saverio Ieva, Agnese Pinto, and Eugenio Di Sciascio. 2014. A mobile matchmaker for the Ubiquitous Semantic Web. International fournal on Semantic Web and Information Systems 10, 4 (dec 2014), 77-100.

[4] W3C OWL Working Group. 2012. OWL 2 Web Ontology Language Document Overview (Second Edition). W3C Recommendation. W3C. https://www.w3.org/TR/owl2-overview/.

[5] Roy Want, Bill N Schilit, and Scott Jenson. 2015. Enabling the Internet of Things. Computer 48, 1 (2015), 28-35.

\footnotetext{
${ }^{10}$ http://sisinflab.poliba.it/swottools/physicalweb/demo-wine.html
} 